\title{
A systematic review of deep brain stimulation for the treatment of drug-resistant epilepsy in childhood
}

\author{
Han Yan, MD, ${ }^{1}$ Eric Toyota, BSc, ${ }^{2}$ Melanie Anderson, MLIS, ${ }^{3}$ Taylor J. Abel, MD, ${ }^{4}$ \\ Elizabeth Donner, MD, ${ }^{5}$ Suneil K. Kalia, MD, PhD, ${ }^{1,6}$ James Drake, MBBCh, MSc, ${ }^{1,7}$ \\ James T. Rutka, MD, PhD, ${ }^{1,7}$ and George M. Ibrahim, MD, PhD ${ }^{1,7}$
}

\begin{abstract}
'Division of Neurosurgery, Department of Surgery, University of Toronto; 'Queen's School of Medicine, Queen's University, Kingston; ${ }^{~}$ Library and Information Services, University Health Network, University of Toronto; ${ }^{5}$ Division of Neurology, The Hospital for Sick Children, Toronto; ${ }^{6}$ Division of Neurosurgery, Toronto Western Hospital, Toronto; ${ }^{7}$ Division of Neurosurgery, The Hospital for Sick Children, Toronto, Ontario, Canada; and ${ }^{4}$ Department of Neurosurgery, University of Pittsburgh, Pennsylvania
\end{abstract}

OBJECTIVE Drug-resistant epilepsy (DRE) presents a therapeutic challenge in children, necessitating the consideration of multiple treatment options. Although deep brain stimulation (DBS) has been studied in adults with DRE, little evidence is available to guide clinicians regarding the application of this potentially valuable tool in children. Here, the authors present the first systematic review aimed at understanding the safety and efficacy of DBS for DRE in pediatric populations, emphasizing patient selection, device placement and programming, and seizure outcomes.

METHODS The systematic review was conducted according to the Preferred Reporting Items for Systematic Reviews and Meta-Analyses (PRISMA) guidelines and recommendations. Relevant articles were identified from 3 electronic databases (MEDLINE, Embase, and Cochrane CENTRAL) from their inception to November 17, 2017. Inclusion criteria of individual studies were 1) diagnosis of DRE; 2) treatment with DBS; 3 ) inclusion of at least 1 pediatric patient (age $\leq$ 18 years); and 4) patient-specific data. Exclusion criteria for the systematic review included 1) missing data for age, DBS target, or seizure freedom; 2) nonhuman subjects; and 3) editorials, abstracts, review articles, and dissertations.

RESULTS This review identified 21 studies and 40 unique pediatric patients (ages 4-18 years) who received DBS treatment for epilepsy. There were 18 patients with electrodes placed in the bilateral or unilateral centromedian nucleus of the thalamus (CM) electrodes, 8 patients with bilateral anterior thalamic nucleus (ATN) electrodes, 5 patients with bilateral and unilateral hippocampal electrodes, 3 patients with bilateral subthalamic nucleus (STN) and 1 patient with unilateral STN electrodes, 2 patients with bilateral posteromedial hypothalamus electrodes, 2 patients with unilateral mammillothalamic tract electrodes, and 1 patient with caudal zona incerta electrode placement. Overall, 5 of the $40(12.5 \%)$ patients had an International League Against Epilepsy class I (i.e., seizure-free) outcome, and 34 of the 40 (85\%) patients had seizure reduction with DBS stimulation.

CONCLUSIONS DBS is an alternative or adjuvant treatment for children with DRE. Prospective registries and future clinical trials are needed to identify the optimal DBS target, although favorable outcomes are reported with both CM and ATN in children.

https://thejns.org/doi/abs/10.3171/2018.9.PEDS18417

KEYWORDS DBS; epilepsy; children; functional neurosurgery

$\mathrm{D}$ RUG-RESISTANT epilepsy is a complex condition that may be treated surgically using a wide range of procedures in the appropriately selected patient. These include resections and ablative surgery, functional disconnections, and neuromodulation. In children, surgical treatments are increasingly emphasized given the medical and psychosocial burden of drug-resistant epilepsy (DRE) ${ }^{22}$ and the detrimental effects of seizures and medications on the developing brain. ${ }^{16}$ The Early Randomized Surgical Epilepsy Trial provided class I evidence for the benefit of early resection ${ }^{11}$ compared with medical management in adolescents with temporal lobe epilepsy.

ABBREVIATIONS ATN = anterior thalamic nucleus; $C M=$ centromedian nucleus of the thalamus; $D B S=$ deep brain stimulation; $\mathrm{DRE}=$ drug-resistant epilepsy; RNS = responsive neurostimulation; STN = subthalamic nucleus; VNS = vagus nerve stimulation.

SUBMITTED July 2, 2018. ACCEPTED September 24, 2018.

INCLUDE WHEN CITING Published online November 30, 2018; DOI: 10.3171/2018.9.PEDS18417. 
Furthermore, a single-center randomized controlled trial of children younger than 18 years with DRE demonstrated a $77 \%$ seizure freedom for surgical treatment compared with $7 \%$ for medical treatment. ${ }^{10}$ In patients without localization-related epilepsy or unacceptable iatrogenic risk to eloquent cortex, neuromodulation may decrease the seizure burden and improve quality of life. ${ }^{31}$ Vagus nerve stimulation (VNS) is a viable outcome with long-term seizure reduction, ${ }^{31}$ although complications such as hardware failure, deep infection, hoarseness, dysphasia, and torticollis have been described in children. ${ }^{36}$ The responsive neurostimulation (RNS) system was approved by the FDA in 2003 for use in patients 18 years or older with DRE. Studies have demonstrated that adults can benefit from moderate seizure reduction, ${ }^{14,17}$ but this new therapy has not yet been thoroughly studied in children.

Deep brain stimulation (DBS) is a therapeutic option that delivers electrical stimulation in order to modulate cortical excitability, thereby reducing the frequency and severity of seizures in an adjustable and reversible manner. The SANTE (Stimulation of the Anterior Nucleus of the Thalamus for Epilepsy) trial demonstrated statistically significant reductions in seizure frequency in a multicenter prospective randomized cohort of 110 adults with DRE who underwent anterior nucleus DBS. ${ }^{12} \mathrm{Sev}-$ eral DBS targets have been studied, ${ }^{8}$ including the anterior thalamic nucleus (ATN) for patients with frontotemporal epilepsy, ${ }^{21,27,32}$ the centromedian nucleus of the thalamus (CM) for patients with generalized epilepsy, ${ }^{40,43,45}$ and the hippocampus for patients with temporal lobe epilepsy.,6

DBS for children is often only considered when patients have reached a treatment-refractory stage of their disease, often with few options remaining. ${ }^{30}$ Most commonly used to treat intractable primary generalized childhood dystonia, the potential for DBS in pediatric populations offers new hope to improve a child's quality of life. Despite its potential value, there remain important unanswered questions regarding DBS for epilepsy in children. Current evidence is limited to case reports and small case series; long-term data regarding its safety and efficacy are lacking. Much of the evidence is translated from adult studies; the procedure and its associated risks as well as device programming and follow-up are modified, thereby posing a challenge to clinicians given the biological differences in children. Although DBS could provide significant seizure freedom for children with DRE, it is not offered routinely, as it is still fairly novel in pediatric populations. The current report is the first to present a synthesis of the available evidence for DBS in children with DRE to assess the efficacy and safety of DBS in pediatric patients with DRE. This systematic review aims to analyze the current literature to understand the effects of DBS for epilepsy in a pediatric population, focusing on safety and efficacy and highlighting patient selection, DBS placement and settings, and seizure freedom.

\section{Methods}

\section{Search Strategy}

This systematic review was conducted according to the Preferred Reporting Items for Systematic Reviews and Meta-Analyses (PRISMA) ${ }^{28}$ guidelines and recommendations. The strategy was developed a priori but not published. A literature search was performed using MEDLINE, Embase, and Cochrane CENTRAL on November 17, 2017, by a librarian (M.A.). The database searches used keywords (individually and/or in combination), specifically "electrical stimulation," "deep brain stimulation" or "DBS," and "seizure(s)," or "epilepsy" with the appropriate subject headings. The reference lists of retrieved review articles were reviewed to identify additional relevant articles.

\section{Study Selection and Data Extraction}

Retrieved studies were systematically assessed using inclusion and exclusion criteria by 2 reviewers (H.Y. and E.T.). Inclusion criteria were 1) diagnosis of DRE, as defined by the individual studies; 2) treatment with DBS; 3) inclusion of at least 1 pediatric patient; and 4) patient-specific data. Exclusion criteria for the systematic review included 1) missing data for age, DBS target, or seizure freedom; 2) nonhuman subjects; and 3) editorials, abstracts, review articles, and dissertations. When duplicate studies were found, only the most recent and complete reports were included for quantitative assessment.

All data were extracted from article texts, tables, and figures. Each retrieved article was reviewed by 2 investigators independently (H.Y. and E.T.). Any discrepancies were reviewed in conference.

\section{Results Literature Search}

The search strategy identified a total of 6352 studies (Fig. 1). After removal of 1490 duplicate studies, inclusion and exclusion criteria were applied to the titles of the 4862 articles. This yielded 26 studies that underwent full-text analysis, of which 5 studies did not meet the inclusion criteria (Fig. 1). When patients originated from the same hospital, the demographic information of the patients was analyzed. Six patients were included in multiple papers, $, 27,33,40,42,44$ and only the most recent publication was included in this analysis. Thus, 21 studies were included.

\section{Cohort Description}

A total of 40 patients were included in this systematic review of pediatric epilepsy patients treated with DBS (Table 1). The ages ranged from $4^{40}$ to 18 years. Sex was not reported for 10 patients. ${ }^{40,44}$ Of the remaining 30 patients, there were 19 males and 11 females. The shortest follow-up duration was 0.5 months, ${ }^{44}$ and this was in the context of a protocol to follow DBS of the hippocampus with a subsequent temporal lobectomy. The majority of patients $(n=24)$ had at least 18 months of follow-up when seizure freedom was measured, although 2 patients only had reported follow-up of 2 weeks.

\section{Seizure Characteristics}

All patients included were offered DBS treatment because their epilepsy was refractory to medical treatment. These patients had a wide range of etiologies ranging from focal epilepsy secondary to focal cortical dysplasia 


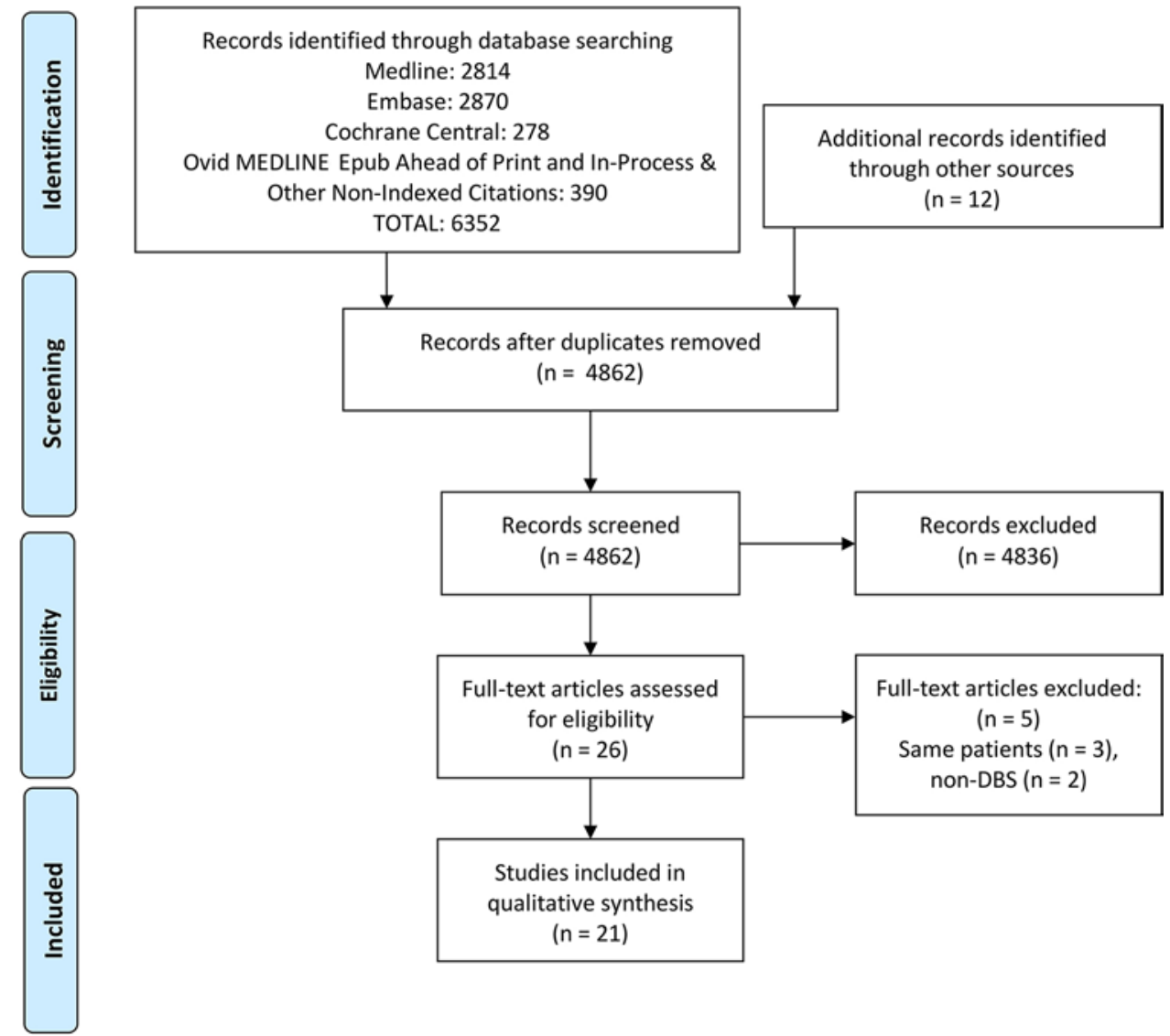

FIG. 1. PRISMA flowchart. Figure is available in color online only.

to multifocal epilepsy resulting from genetic or syndromic conditions. Two patients were previously diagnosed with tuberous sclerosis, ${ }^{3,40} 2$ patients had mesial temporal sclerosis, ${ }^{7,41} 2$ had hypothalamic hamartomas, ${ }^{18}$ and 3 had cortical dysplasia. ${ }^{2,5,20}$

One patient included in the analysis was one of 3 individuals from a family with North Sea progressive myoclonus epilepsy and confirmed GOSR 2 mutation. ${ }^{1}$ All 3 members of this family presented with ataxia, tremor, early gait difficulties, and myoclonic and generalized tonic-clonic epilepsy, and each received benefit from DBS treatment; only 1 of these patients was a child. Kokoszka et al. ${ }^{20}$ presented a patient with West syndrome who, historically as an infant, had classic findings of infantile spasms with the electroencephalogram having shown hypsarrhythmia. Another child had been diagnosed with autosomal-dominant nocturnal frontal lobe epilepsy that did not respond to DBS treatment. ${ }^{5}$ Nine patients from the same study all had Lennox-Gastaut syndrome, ${ }^{40}$ a childhood epilepsy pathology characterized by drug-resistant generalized seizures and cognitive deterioration. These seizures were associated with slow spike-wave complexes and bursts of rapid rhythms during slow sleep on electroencephalography.

Seizure type was described in a heterogeneous manner by the different studies. The majority of patients $(n=$ 27) had generalized or secondarily generalized seizures (Table 2). Four patients had previous episodes of status epilepticus, ${ }^{7,24,45}$ including 1 patient who was in a continu- ous 4-week episode of intractable status epilepticus, which only improved after DBS treatment. ${ }^{24}$

Five patients had previous surgery in an attempt to treat their epilepsy. $2,5,9,20,37$ The operations included a biopsy to identify a possible epileptogenic lesion, ${ }^{2}$ a right precentral frontal lobe resection ${ }^{5}$ and a left anterior temporal lobectomy ${ }^{9}$ to attempt seizure reduction, and $\mathrm{VNS}^{0}{ }^{37} 1$ patient had already undergone right fontal lobectomy, complete corpus callosotomy, VNS, left temporal lobectomy, and posterior quandrantectomy. ${ }^{20}$ These procedures did not achieve the desired seizure reduction, and DBS was offered as an alternative treatment. Two patients had subacute electrical stimulation of the hippocampal formation or gyrus for 2-3 weeks to identify and suppress temporal lobe epileptogenesis prior to temporal lobectomy. ${ }^{41}$ In the 16 days of monitoring, DBS treatment eliminated seizures for one of these patients. Given the complex and dissimilar histories of these patients, it is difficult to correlate specific risk factors that would predict the effectiveness of surgery or DBS.

Since all patients included in this study were diagnosed with DRE, these children were receiving at a minimum 2 seizure medications, and 1 child had been on 6 seizure medications simultaneously prior to DBS surgery. ${ }^{24}$ Although most of the studies did not report post-DBS medication changes, all studies that reported these data in tables or text demonstrated a decrease in antiepileptic medication. ${ }^{3,29,40,45}$ 
TABLE 1. Demographic information

\begin{tabular}{|c|c|c|c|c|c|}
\hline Authors \& Year & Country & Pt No. & Sex & Age (yrs) & Notes \\
\hline Anderson et al., 2017 & South Africa & 1 & M & 9 & North Sea progressive myoclonus epilepsy w/ GOSR2 mutation \\
\hline Benabid et al., 2002 & France & 2 & $\mathrm{~F}$ & 5 & Cortical dysplasia \\
\hline \multirow{2}{*}{ Benedetti-Isaac et al., 2015} & \multirow{2}{*}{ Colombia } & 3 & M & 16 & Tuberous sclerosis \\
\hline & & 4 & M & 16 & Autism \\
\hline \multirow{2}{*}{ Chabardès et al., 2002} & \multirow{2}{*}{ France } & 5 & M & 9 & Focal cortical dysplasia; infection \& temporary unilat battery removal \\
\hline & & 6 & M & 17 & \\
\hline Cukiert et al., $2017^{7}$ & Brazil & 7 & $\mathrm{~F}$ & 14 & Lt mesial temp sclerosis, recurrent status epilepticus \\
\hline Ding et al., 2016 & China & 8 & M & 10 & Febrile convulsive seizures \\
\hline Fisher et al., 1992 & US & 9 & M & 16 & \\
\hline \multirow{2}{*}{ Khan et al., 2009} & \multirow{2}{*}{ UK } & 10 & M & 16 & Rt hypothalamic hamartoma \\
\hline & & 11 & $\mathrm{~F}$ & 13 & Lt hypothalamic hamartoma \\
\hline Kim et al., 2017 & South Korea & 12 & $\mathrm{~F}$ & 16 & \\
\hline Kokoszka et al., 2018 & US & 13 & M & 14 & Type 1 focal cortical dysplasia \\
\hline Lee et al., 2017 & Taiwan & 14 & $\mathrm{~F}$ & 17 & 4 wks of status epilepticus prior to DBS \\
\hline \multirow{2}{*}{ Lee et al., 2006} & \multirow{2}{*}{ South Korea } & 15 & M & 14 & Removal due to infection \\
\hline & & 16 & $\mathrm{~F}$ & 14 & \\
\hline \multirow{3}{*}{ Lee et al., 2012} & \multirow{3}{*}{ South Korea } & 17 & $\mathrm{~F}$ & 14 & \\
\hline & & 18 & M & 14 & \\
\hline & & 19 & M & 18 & \\
\hline Lim et al., 2007 & Taiwan & 20 & M & 18 & ATN \& STN insertion, removal of STN \\
\hline Valentín et al., 2013 & UK/Spain & 21 & $\mathrm{~F}$ & 18 & \\
\hline \multirow{3}{*}{ Valentín et al., 2017} & \multirow{3}{*}{ UK } & 22 & M & 14 & \\
\hline & & 23 & M & 9 & \\
\hline & & 24 & $\mathrm{~F}$ & 8 & \\
\hline \multirow{3}{*}{ Velasco et al., 1987} & \multirow{3}{*}{ Mexico } & 25 & $\mathrm{~F}$ & 17 & \\
\hline & & 26 & M & 15 & Previous status epilepticus \\
\hline & & 27 & M & 16 & Previous status epilepticus \\
\hline \multirow{2}{*}{ Velasco et al., $2000^{41}$} & \multirow{2}{*}{ Mexico } & 28 & $\mathrm{~F}$ & 11 & Medial temp sclerosis \\
\hline & & 29 & M & 11 & \\
\hline Velasco et al., $2000^{44}$ & Mexico & 30 & & 15 & \\
\hline \multirow{9}{*}{ Velasco et al., 2006} & \multirow{9}{*}{ Mexico } & 31 & & 8 & \\
\hline & & 32 & & 7 & Explanted due to skin erosion \\
\hline & & 33 & & 9 & Explanted due to skin erosion \\
\hline & & 34 & & 13 & \\
\hline & & 35 & & 11 & Rupture of electrode lead, replacement \\
\hline & & 36 & & 10 & \\
\hline & & 37 & & 4 & Tuberous sclerosis \\
\hline & & 38 & & 13 & \\
\hline & & 39 & & 13 & Cerebral infarct \\
\hline Velasco et al., $2007^{42}$ & Mexico & 40 & $M$ & 14 & \\
\hline
\end{tabular}

$\mathrm{Pt}=$ patient; temp = temporal; UK = United Kingdom.

\section{DBS Location and Seizure Freedom}

The DBS target was determined largely by the institution and the experience of the surgeon treating adult populations (Table 3). Seizure reduction was heterogeneously reported, mostly by self-report at follow-up appointments; only 1 study $^{7}$ clarified the use of a seizure diary. Ultimately, 5 of the 40 patients $(12.5 \%)$ had an International League Against Epilepsy class I (i.e., seizure-free) out- come. Among the 40 patients, 34 (85\%) patients had seizure reduction with DBS stimulation, and $6(15 \%)$ patients had no seizure reduction.

There were 18 patients from 7 different studies who had DBS electrodes placed in the CM bilaterally or unilaterally. ${ }^{13,19,37,38,40,44,45}$ There was seizure reduction in 17 of these patients, ranging from $30 \%$ to $100 \%$. There were 9 patients with Lennox-Gastaut syndrome who were treated 
TABLE 2. Epilepsy characteristics

\begin{tabular}{|c|c|c|c|c|c|c|c|}
\hline Authors \& Year & $\begin{array}{l}\text { Pt } \\
\text { No. }\end{array}$ & $\begin{array}{l}\text { Duration } \\
\text { of } \\
\text { Epilepsy } \\
\text { (mos) }\end{array}$ & $\begin{array}{l}\text { Sz } \\
\text { Frequency } \\
\text { (no./mo) }\end{array}$ & Localization & Syndrome & Antiepileptic Agents & Other Treatment \\
\hline $\begin{array}{l}\text { Anderson et al., } \\
2017\end{array}$ & 1 & 36 & 6 & Generalized & $\begin{array}{l}\text { North Sea progres- } \\
\text { sive myoclonus } \\
\text { epilepsy }\end{array}$ & $\begin{array}{l}\text { Carbamazepine, sodium } \\
\quad \text { valproate }\end{array}$ & \\
\hline $\begin{array}{l}\text { Benabid et al., } \\
\quad 2002\end{array}$ & 2 & 36 & 210 & Lt parietal & NA & $\begin{array}{l}\text { Phenytoin, carbamazepine, } \\
\text { stiripentol, } \gamma \text {-vinyl-GABA, clo- } \\
\text { bazam; pre-Sx phenobarbi- } \\
\text { tal, valproic acid, clonazepam }\end{array}$ & Previous It parietal biopsy \\
\hline \multirow{2}{*}{$\begin{array}{l}\text { Benedetti-Isaac } \\
\text { et al., } 2015\end{array}$} & 3 & 188 & 1200 & Generalized & NA & Carbamazepine, clonazepam & \\
\hline & 4 & 132 & 12 & Generalized & NA & $\begin{array}{l}\text { Phenytoin, clonazepam, } \\
\text { lorazepam }\end{array}$ & \\
\hline \multirow{2}{*}{$\begin{array}{l}\text { Chabardès et al., } \\
2002\end{array}$} & 5 & 103 & 245 & Rt central & NA & Valproate, lamotrigine & Rt precentral frontal resection \\
\hline & 6 & 132 & 600 & Lt insulofrontal & ADNFLE & $\begin{array}{l}\text { Phenytoin, clonazepam, leveti- } \\
\text { racetam, piracetam }\end{array}$ & \\
\hline $\begin{array}{l}\text { Cukiert et al., } \\
\qquad 2017^{7}\end{array}$ & 7 & 132 & 10 & Lt temp & NA & NA & \\
\hline Ding et al., 2016 & 8 & 126 & NA & Generalized & NA & NA & $\begin{array}{l}\text { Previous It anterior temp } \\
\text { lobectomy }\end{array}$ \\
\hline Fisher et al., 1992 & 9 & 168 & 30 & Generalized & NA & Phenytoin, clorazepate & \\
\hline \multirow[b]{2}{*}{ Khan et al., 2009} & 10 & 96 & 12 & Generalized & NA & Multiple & Refused resective surgery \\
\hline & 11 & 141 & 120 & $\begin{array}{l}\text { Gelastic, gen- } \\
\text { eralized }\end{array}$ & NA & NA & \\
\hline Kim et al., 2017 & 12 & 180 & NA & $\begin{array}{l}\text { Bilat frontal } \\
\text { parietal }\end{array}$ & NA & NA & \\
\hline $\begin{array}{l}\text { Kokoszka et al., } \\
2018\end{array}$ & 13 & 162 & NA & Lt temp & West syndrome & NA & $\begin{array}{l}\text { Concurrent RNS; previous } \\
\text { rt frontal lobectomy, com- } \\
\text { plete corpus callosotomy, } \\
\text { VNS, It temp lobectomy, \& } \\
\text { posterior quandrantectomy }\end{array}$ \\
\hline Lee et al., 2017 & 14 & 60 & 77 & Generalized & NA & $\begin{array}{l}\text { Lorazepam, levetiracetam, } \\
\text { valproic acid, topiramate, } \\
\text { perampanel, midazolam }\end{array}$ & $\begin{array}{l}\text { Ketogenic diet, midazolam } \\
\text { infusion }\end{array}$ \\
\hline \multirow[t]{2}{*}{ Lee et al., 2006} & 15 & NA & 42 & Generalized & $\begin{array}{l}\text { Global cognitive } \\
\text { delay }\end{array}$ & $\begin{array}{l}\text { Topiramate, lamotrigine, valpro- } \\
\text { ate, vigabatrin }\end{array}$ & \\
\hline & 16 & NA & 450 & Rt motor & NA & NA & \\
\hline \multirow{3}{*}{ Lee et al., 2012} & 17 & 36 & 1200 & Generalized & NA & NA & \\
\hline & 18 & 60 & 95 & Bilat frontal & NA & NA & \\
\hline & 19 & 84 & 30 & $\begin{array}{l}\text { Bilat centropa- } \\
\text { rietal }\end{array}$ & NA & NA & \\
\hline Lim et al., 2007 & 20 & 174 & 26 & Generalized & NA & $\begin{array}{l}\text { Carbamazepine, topiramate, } \\
\text { clonazepam }\end{array}$ & \\
\hline $\begin{array}{l}\text { Valentín et al., } \\
2013\end{array}$ & 21 & 168 & 3006 & Generalized & NA & $\begin{array}{l}\text { Levetiracetam, lamotrigine, } \\
\text { acetazolamide, clonazepam }\end{array}$ & Previous VNS \\
\hline \multirow{3}{*}{$\begin{array}{l}\text { Valentín et al., } \\
2017\end{array}$} & 22 & NA & 10 & Focal motor & NA & NA & \\
\hline & 23 & NA & $>1000$ & Generalized & NA & NA & \\
\hline & 24 & NA & 900 & Generalized & $\begin{array}{l}\text { Genetic syndrome } \\
\text { NYD }\end{array}$ & NA & \\
\hline
\end{tabular}




\begin{tabular}{|c|c|c|c|c|c|c|c|}
\hline Authors \& Year & $\begin{array}{l}\text { Pt } \\
\text { No. }\end{array}$ & $\begin{array}{c}\text { Duration } \\
\text { of } \\
\text { Epilepsy } \\
\text { (mos) }\end{array}$ & $\begin{array}{c}\mathrm{Sz} \\
\text { Frequency } \\
\text { (no./mo) }\end{array}$ & Localization & Syndrome & Antiepileptic Agents & Other Treatment \\
\hline \multirow{3}{*}{$\begin{array}{l}\text { Velasco et al., } \\
1987\end{array}$} & 25 & & $10-37$ & Generalized & NA & $\begin{array}{l}\text { Phenytoin, carbamazepine, } \\
\text { valproate }\end{array}$ & AED overdose \\
\hline & 26 & & $7-150$ & Generalized & NA & $\begin{array}{l}\text { Phenytoin, carbamazepine, } \\
\text { clonazepam }\end{array}$ & AED overdose \\
\hline & 27 & & $26-60$ & Generalized & NA & $\begin{array}{l}\text { Primidone, carbamazepine, } \\
\text { clonazepam }\end{array}$ & \\
\hline \multirow{2}{*}{$\begin{array}{l}\text { Velasco et al., } \\
2000^{41}\end{array}$} & 28 & 72 & 4 & Focal & NA & Oxcarbazepine & Post-DBS temp lobectomy \\
\hline & 29 & 48 & 12 & Generalized & NA & Carbamazepine, valproate & Post-DBS temp lobectomy \\
\hline $\begin{array}{l}\text { Velasco et al., } \\
2000^{44}\end{array}$ & 30 & NA & 119 & Generalized & NA & NA & \\
\hline \multirow{9}{*}{$\begin{array}{l}\text { Velasco et al., } \\
2006\end{array}$} & 31 & 12 & 3119 & Generalized & Lennox-Gastaut & NA & \\
\hline & 32 & 12 & 4300 & Generalized & Lennox-Gastaut & NA & \\
\hline & 33 & 24 & 3780 & Generalized & Lennox-Gastaut & NA & \\
\hline & 34 & 96 & 3030 & Generalized & Lennox-Gastaut & NA & \\
\hline & 35 & 108 & 1200 & Generalized & Lennox-Gastaut & NA & \\
\hline & 36 & 115 & 50 & Generalized & Lennox-Gastaut & NA & \\
\hline & 37 & 46 & 150 & Atypical & Lennox-Gastaut & NA & \\
\hline & 38 & 108 & 35 & Generalized & Lennox-Gastaut & NA & \\
\hline & 39 & 72 & 50 & Atypical & Lennox-Gastaut & NA & \\
\hline $\begin{array}{l}\text { Velasco et al., } \\
2007^{42}\end{array}$ & 40 & 36 & 25 & Generalized & & Carbamazepine, phenytoin & \\
\hline
\end{tabular}

ADNFLE = autosomal-dominant nocturnal frontal lobe epilepsy; $A E D=$ antiepileptic drug; NA = not available; NYD = not yet diagnosed; $S x=$ symptom(s); $\mathrm{Sz}=\operatorname{seizure}$.

with unilateral or bilateral CM DBS. ${ }^{40}$ This study included the 5 patients with unilateral CM electrode placement and stimulation, due to inaccurate placement of one of 2 bilateral CM electrodes.

Eight patients from 6 different studies had DBS electrodes placed in the anterior thalamic nucleus (ATN) bilaterally. ${ }^{20,24-26,29,38}$ There was seizure reduction in 6 of the 8 patients, with seizure reduction ranging from $37 \%$ to $90 \%$. The 2 patients with no improvement in seizure outcome had seizures localized to the bilateral frontal lobe and bilateral centroparietal lobe. ${ }^{26}$ The most recent study ${ }^{20}$ uniquely attempted the use of a right temporal cortical strip for RNS and a left thalamic depth electrode simultaneously inserted during the same operation, using an offlabel application of the RNS for children. A corticothalamic stimulation trial showed improved 50\% reduction in seizure frequency with DBS and cortical detection compared with unilateral cortical stimulation from RNS alone.

Five patients had DBS electrodes placed in the hippocampus. ${ }^{7,9,41,42}$ Two of these patients with intractable temporal lobe seizures had bilateral depth and subdural electrodes implanted to determine the location and extent of the epileptic focus before a temporal lobectomy. ${ }^{41}$ These electrodes only provided stimulation for 16 days, with seizure freedom in one patient and no change in the other. Three of the 5 patients were treated with DBS electrode placement in the hippocampus unilaterally. Two patients had left hippocampal DBS placement: one patient had left mesial temporal sclerosis with $0 \%$ seizure rate reduction, ${ }^{7}$ and the other patient was enrolled in a double-blind study where the stimulation was on or off for 1 month following implantation and demonstrated $64 \%$ seizure reduction when the stimulation was on. ${ }^{42}$ The patient with right hippocampal DBS placement had previously undergone left anterior temporal lobectomy, with $80 \%$ seizure reduction.

Three patients had bilateral subthalamic nucleus (STN) DBS placement, in an attempt to inhibit the substantia nigra from spreading paroxysmal discharges to disrupt seizure pathways.,52 One of these 3 patients had successful seizure reduction (71.4\%) after 1 month, but the case was complicated by infection of the implantable pulse generator and the entire system had to be explanted. ${ }^{25}$ Similarly, another patient with focal cortical dysplasia had bilateral STN placement after a failed precentral frontal resection also had an implantable pulse generator infection with a temporary explantation; during this period, the seizure frequency increased. ${ }^{5} \mathrm{~A}$ third patient who was diagnosed with autosomal-dominant nocturnal frontal lobe epilepsy was treated with bilateral STN DBS placement and had no change in seizure frequency. ${ }^{5}$ One child in whom a previous left parietal biopsy had led to the diagnosis of cortical dysplasia underwent left-sided STN placement, with an $80.7 \%$ seizure reduction. ${ }^{2}$ One patient initially underwent insertion of ATN and STN electrodes, but the STN 
TABLE 3. DBS and seizure outcomes

\begin{tabular}{|c|c|c|c|c|c|c|c|c|c|}
\hline Authors \& Year & $\begin{array}{l}\text { Pt } \\
\text { No. }\end{array}$ & $\begin{array}{c}\text { DBS } \\
\text { Location }\end{array}$ & Anesthesia & $\begin{array}{l}\text { Frequency } \\
\quad(\mathrm{Hz})\end{array}$ & $\begin{array}{l}\text { Pulse Width } \\
\text { ( } \mu \mathrm{sec})\end{array}$ & $\begin{array}{l}\text { Volt/ } \\
\text { Current }\end{array}$ & Sz Reduction & $\begin{array}{c}\mathrm{FU} \\
(\mathrm{mos})\end{array}$ & Notes \\
\hline Anderson et al., 2017 & 1 & Bilat cZl & NR & 130 & 450 & $2.1 \mathrm{~mA}$ & $100 \%$ of GTC & 84 & \\
\hline Benabid et al., 2002 & 2 & Lt STN & GA & 130 & 90 & $5.2 \mathrm{~V}$ & $80.7 \%$ & 30 & \\
\hline \multirow[b]{2}{*}{$\begin{array}{l}\text { Benedetti-Isaac et } \\
\quad \text { al., } 2015\end{array}$} & 3 & Bilat pHyp & GA & 185 & 90 & $2.7 \mathrm{~V}$ & $100 \%$ & 48 & Improved aggression \\
\hline & 4 & Bilat pHyp & GA & 185 & 90 & $2.8 \mathrm{~V}$ & $89.6 \%$ & 2 & $\begin{array}{l}\text { Aggression improved } \\
\text { for only } 2 \text { mos }\end{array}$ \\
\hline \multirow{2}{*}{$\begin{array}{l}\text { Chabardès et al., } \\
2002\end{array}$} & 5 & Bilat STN & GA & 130 & 90 & $3.8 \mathrm{~V}$ & $67.8 \%$ & 15 & \\
\hline & 6 & Bilat STN & GA & 130 & 60 & $3.0 \mathrm{~V}$ & $0 \%$ & 6 & \\
\hline Cukiert et al., $2017^{7}$ & 7 & $\begin{array}{l}\text { Lt hippo- } \\
\text { campus }\end{array}$ & GA & 130 & 300 & $2.0 \mathrm{~V}$ & $0 \%$ & $>6$ & \\
\hline Ding et al., 2016 & 8 & $\begin{array}{l}\text { Rt hippo- } \\
\text { campus }\end{array}$ & GA & 130 & 300 & $2.2 \mathrm{~V}$ & $80 \%$ & 18 & \\
\hline Fisher et al., 1992 & 9 & Bilat CM & NR & 65 & 90 & $0.5-10 \mathrm{~V}$ & $\sim 30 \%$ & $>3$ & $\begin{array}{l}50 \% \text { reduction stimula- } \\
\text { tor off, } 15 \% \text { reduc- } \\
\text { tion w/ stimulator on }\end{array}$ \\
\hline \multirow[b]{2}{*}{ Khan et al., 2009} & 10 & Rt MMT & GA & 140 & 90 & $3 \mathrm{~V}$ & $100 \%$ & 21 & \\
\hline & 11 & Lt MMT & GA & 140 & 90 & $3.5 \mathrm{~V}$ & $86 \%$ & 13 & $\begin{array}{l}\text { Returned to school } \\
\text { after } 2 \text { yrs }\end{array}$ \\
\hline Kim et al., 2017 & 12 & Bilat CM & GA & 130 & 90 & $1.5-2.0 \mathrm{~V}$ & $92.9 \%$ & 18 & \\
\hline Kokoszka et al., 2018 & 13 & Bilat ATN & NR & $100 ; 200$ & $160 ; 160$ & $0.5 \mathrm{~mA}$ & $80-90 \%$ & 19 & $\begin{array}{l}\text { Responsive neurostim- } \\
\text { ulation also used }\end{array}$ \\
\hline Lee et al., 2017 & 14 & Bilat ATN & NR & 145 & 120 & $8 \mathrm{~V}$ & $90 \%$ & 1.5 & \\
\hline \multirow{2}{*}{ Lee et al., 2006} & 15 & Bilat STN & Local & 130 & 90 & NA & $71.4 \%$ & 1 & \\
\hline & 16 & Bilat ATN & Local & 130 & 90 & NA & $50 \%$ & 2 & \\
\hline \multirow{3}{*}{ Lee et al., 2012} & 17 & Bilat ATN & Local & 100-185 & $90-150$ & $1.5-3.1 \mathrm{~V}$ & $80.8 \%$ & 59 & \\
\hline & 18 & Bilat ATN & Local & $100-185$ & $90-150$ & $1.5-3.1 \mathrm{~V}$ & $0 \%$ & 24 & \\
\hline & 19 & Bilat ATN & Local & 100-185 & $90-150$ & $1.5-3.1 \mathrm{~V}$ & $0 \%$ & 28 & \\
\hline Lim et al., 2007 & 20 & Bilat ATN & Local & 180 & 90 & $6 \mathrm{~V}$ & $37 \%$ & 48 & \\
\hline Valentín et al., 2013 & 21 & Bilat CM & GA & 60 & 90 & $5 \mathrm{~V}$ & $\begin{array}{l}50 \% \text { for complex partial; } \\
95 \% \text { for simple partial }\end{array}$ & 36 & \\
\hline \multirow{3}{*}{ Valentín et al., 2017} & 22 & Bilat ATN & GA & NA & NA & NA & $>60 \%$ & 12 & \\
\hline & 23 & Bilat CM & GA & NA & NA & NA & $>60 \%$ & 48 & \\
\hline & 24 & Bilat CM & GA & NA & NA & NA & $0 \%$ & 18 & \\
\hline \multirow{3}{*}{ Velasco et al., 1987} & 25 & Bilat CM & NR & $60-100$ & 100 & $2.0 \mathrm{~mA}$ & $\begin{array}{l}85 \% \text { for tonic-clonic; } \\
100 \% \text { for complex } \\
\text { partial }\end{array}$ & 3 & $\begin{array}{l}\text { Reduced AED dos- } \\
\text { ages \& side effects }\end{array}$ \\
\hline & 26 & Bilat CM & NR & $60-100$ & 100 & $2.0 \mathrm{~mA}$ & $\begin{array}{l}100 \% \text { for tonic-clonic, } \\
\text { complex partial }\end{array}$ & 3 & $\begin{array}{l}\text { Reduced AED dos- } \\
\text { ages \& side effects }\end{array}$ \\
\hline & 27 & Bilat CM & NR & $60-100$ & 100 & $2.0 \mathrm{~mA}$ & $\begin{array}{l}95 \% \text { for tonic-clonic; } \\
100 \% \text { for complex } \\
\text { partial }\end{array}$ & 3 & \\
\hline \multirow{2}{*}{ Velasco et al., $2000^{41}$} & 28 & $\begin{array}{l}\text { Bilat hippo- } \\
\text { campus }\end{array}$ & NR & 130 & 450 & $2-4 \mathrm{~mA}$ & $0 \%$ & 0.5 & \\
\hline & 29 & $\begin{array}{l}\text { Bilat hippo- } \\
\text { campus }\end{array}$ & NR & 130 & 450 & $2-4 \mathrm{~mA}$ & $100 \%$ & 0.5 & \\
\hline Velasco et al., $2000^{44}$ & 30 & Bilat CM & GA & 60 & NA & $4-6 \mathrm{~V}$ & $80.60 \%$ & $>12$ & \\
\hline \multirow{5}{*}{ Velasco et al., 2006} & 31 & Lt CM & GA & 130 & 450 & $6-8 \mathrm{~V}$ & $100 \%$ & 18 & \\
\hline & 32 & Bilat CM & GA & 130 & 450 & $6-8 \mathrm{~V}$ & $100 \%$ & 18 & \\
\hline & 33 & Rt CM & GA & 130 & 450 & $6-8 \mathrm{~V}$ & $95 \%$ & 18 & \\
\hline & 34 & Bilat CM & GA & 130 & 450 & $6-8 \mathrm{~V}$ & $95 \%$ & 18 & \\
\hline & 35 & Bilat CM & GA & 130 & 450 & $6-8 \mathrm{~V}$ & $95 \%$ & 18 & \\
\hline
\end{tabular}


» CONTINUED FROM PAGE 280

TABLE 3. DBS and seizure outcomes

\begin{tabular}{|c|c|c|c|c|c|c|c|c|c|}
\hline Authors \& Year & $\begin{array}{l}\mathrm{Pt} \\
\text { No. }\end{array}$ & $\begin{array}{c}\text { DBS } \\
\text { Location }\end{array}$ & Anesthesia & $\begin{array}{c}\text { Frequency } \\
(\mathrm{Hz})\end{array}$ & $\begin{array}{l}\text { Pulse Width } \\
\text { ( } \mu \mathrm{sec})\end{array}$ & $\begin{array}{c}\text { Volt/ } \\
\text { Current }\end{array}$ & Sz Reduction & $\begin{array}{c}\mathrm{FU} \\
\text { (mos) }\end{array}$ & Notes \\
\hline \multirow{4}{*}{$\begin{array}{l}\text { Velasco et al., } 2006 \\
\text { (continued) }\end{array}$} & 36 & Bilat CM & GA & 130 & 450 & $6-8 \mathrm{~V}$ & $70 \%$ & 18 & \\
\hline & 37 & Rt CM & GA & 130 & 450 & $6-8 \mathrm{~V}$ & $58 \%$ & 18 & \\
\hline & 38 & Rt CM & GA & 130 & 450 & $6-8 \mathrm{~V}$ & $53 \%$ & 18 & \\
\hline & 39 & Lt CM & GA & 130 & 450 & $6-8 \mathrm{~V}$ & $30 \%$ & 18 & \\
\hline Velasco et al., $2007^{42}$ & 40 & $\begin{array}{l}\text { Lt hippo- } \\
\text { campus }\end{array}$ & NR & 130 & 450 & $3.0 \mathrm{~mA}$ & $64 \%$ & 18 & \\
\hline
\end{tabular}

cZI = caudal zona incerta; FU = follow-up; GA = general anesthesia; GTC = generalized tonic-clonic; $M$ MT = mammillothalamic tract; NR = not reported; pHyp = posteromedial hypothalamus; Volt $=$ voltage .

electrode was explanted after the ATN electrode proved to provide better seizure relief. ${ }^{29}$

Two patients underwent bilateral posteromedial hypothalamus DBS in an attempt to treat both epilepsy and aggression. ${ }^{3}$ Both patients had improved seizure control, with $89.6 \%$ and $100 \%$ seizure frequency reduction. The aggression for both patients also improved, although the improvement in 1 patient was only temporary for 2 months.

Two patients with hypothalamic hamartomas were treated with unilateral mammillothalamic tract DBS; both patients had favorable results with seizure reduction of $86 \%$ and $100 \% .^{18}$

Finally, 1 patient with North Sea progressive myoclonus epilepsy underwent bilateral caudal zona incerta DBS placement and showed $100 \%$ reduction in seizures at 84 months. ${ }^{1}$ This treatment was also effective for 2 adult relatives with the same pathology due to genetic mutation of GOSR2.

\section{Complications}

The majority of studies noted very few or no complications, and there were no deaths. There were 4 complications, all due to infection. One patient required an explant of the DBS due to infection of the anterior chest battery with Staphylococcus aureus. ${ }^{25}$ There were also 2 occurrences of skin erosion of batteries in children 7 and 9 years old, leading to explantation. ${ }^{40}$ The second child retained the seizure freedom after DBS explantation. Finally, 1 patient had electrode lead breakage after 31 months, and the battery and electrodes were replaced, with the same favorable seizure outcome..$^{40}$

\section{Discussion}

In this systematic review of pediatric cases of epilepsy treated with DBS, 40 patients with DRE from 21 papers were analyzed. There were 18 patients with bilateral or unilateral CM electrodes, ${ }^{13,19,37,38,40,44,45} 8$ patients with bilateral ATN electrodes, ${ }^{20,24-26,29,38} 3$ patients with unilateral hippocampal electrodes, $, 9,422$ patients with bilateral hippocampal electrodes, ${ }^{41} 3$ patients with bilateral ${ }^{5,25}$ and 1 patient with unilateral STN electrodes, ${ }^{2} 2$ patients with bilateral posteromedial hypothalamus electrodes, ${ }^{3} 2$ patients with unilateral mammillothalamic tract electrodes,${ }^{18}$ and 1 patient with caudal zona incerta electrode placement. ${ }^{1}$
In summary, 34 of the $40(85 \%)$ patients had reduction in seizure frequency with DBS stimulation.

Only 6 patients demonstrated no reduction in seizure frequency. ${ }^{5,7,26,38,41}$ Two of these patients had bilateral ATN placement for seizures identified in the bilateral frontal hemispheres and bilateral centroparietal lobes. ${ }^{26} \mathrm{~A}$ patient with autosomal-dominant nocturnal frontal lobe epilepsy who showed seizures originating from the left insulofrontal cortex did not have any seizure improvement with bilateral STN DBS. ${ }^{5}$ Another nonresponder was an 8-year-old girl with a genetic syndrome of unknown etiology. ${ }^{38} \mathrm{Her}$ bilateral CM DBS treatment was unsuccessful at 12 months with a slight worsening of seizure frequency, and thus the DBS electrodes were removed at 18 months. The patient with bilateral hippocampal DBS placement prior to temporal lobectomy who demonstrated no response was the only participant without a positive response to treatment in the study. ${ }^{41}$ This may have been because this patient had stimulation contacts located in the white matter adjacent to the hippocampus instead of the hippocampus proper. The last nonresponder was the only nonresponder in a prospective, controlled, randomized, double-blind study looking at hippocampal DBS in refractory temporal lobe epilepsy. This 14-year-old girl with left mesial temporal sclerosis demonstrated no change in her simple partial seizures and an increase in her complex partial seizures after left hippocampal DBS.

In this cohort, 16 patients ${ }^{40-42,44,45}$ were from Mexico, 13 of whom had DBS placement in the CM. The earliest study was from $1987^{45}$ and drew on the hypothesis that the red nucleus, and the CM situated above it, can induce cortical desynchronization and block epileptic synchronous discharges. Since the SANTE trial demonstrated 38\% seizure reduction compared with $14.5 \%$ in a multicenter, randomized, double-blinded parallel-group study, the ATN has become the most effective and acceptable therapy for refractory epilepsy. ${ }^{13,35}$ Although DRE is heterogeneous in clinical presentation, certain brain networks may be more important for cortical synchronization and seizure propagation, thus making nodes of these networks more important neuromodulation targets in epilepsy. For example, the cortico-striato-thalamic network and the limbic circuit of Papez have been postulated as targets for stimulation. ${ }^{23,34}$ One hypothesis for the mechanism of ATN DBS is the modulation of ipsilateral Papez structures such as the en- 
torhinal cortex, hippocampus, parahippocampal gyrus, mammillothalamic tract, cingulate, and inferior temporal gyrus. ${ }^{15,23,39}$

Even in children, DBS is now used in conjunction with other treatments. Kokoszka et al..$^{20}$ used RNS as an adjunct to DBS, providing a reversible and modulatory treatment option that provides a capacity for chronic recording of brain activity to better localize seizure foci. Their treatment in a nonambulatory, nonverbal 14-year-old boy not only decreased seizure frequency and severity, but also improved his behavior, attentiveness, and level of engagement at school. This boy's seizures did not respond to antiepileptic medications, right frontal lobectomy, complete corpus callosotomy, VNS, left temporal lobectomy, or posterior quandrantectomy. At 19 months' follow-up, cortical stimulation resulted in sustained reduction in both seizure frequency and severity and subsequent DBS reduced seizure frequency by another $50 \%$. This paper is not a direct comparison between DBS and RNS, as the patient in whom both modalities were used had a complex resection history and possibly unique neural circuitry. Velasco et al. ${ }^{41}$ also utilized hippocampal DBS as an adjunct to temporal lobectomy. Taking patients completely off antiepileptics, in correctly placed hippocampal DBS electrodes, continuous high-frequency and low-intensity stimulation of the anterior pes hippocampi and parahippocampal gyrus close to the amygdaloid nucleus and entorhinal cortex increases seizure threshold and decreases clinical seizures. They also conducted a histopathological study following the temporal lobectomy, showing no difference between the stimulated or nonstimulated hippocampus, suggesting that the DBS mechanisms affect physiology independent of tissue pathology.

The specific indications for DBS for the treatment of childhood epilepsy have yet to be defined. It is often trialed after failed ablative surgery if there is too much risk for resective surgery or the target for resective surgery is unclear. There are no studies that have compared DBS to other neuromodulatory systems, such as RNS or VNS.

Given that the majority of studies included both adult and pediatric patients, the complications specific to children were not thoroughly analyzed and synthesized in this paper. In this series, there were 4 patients who required permanent or temporary explantation due to infection or skin erosion..$^{5,25,40}$ One patient required DBS electrode replacement after a lead was broken..$^{40}$ The youngest patient in this cohort was 4 years old, and it is currently unreported if younger children would benefit from DBS for DRE. The long-term complications of DBS in children is not yet understood, although one may hypothesize of unknown migration challenges with placing a DBS electrode in a growing brain.

Limitations of this study include the inclusion of only 1 randomized control trial, 5 blinded studies, ${ }^{13,29,37,42,44}$ and 8 prospective trials, $, 13,19,26,29,37,40-42,44$ with the remaining 12 studies being retrospective case reports and case series. Furthermore, it is difficult to analyze and compare the effectiveness of DBS between studies, as different metrics and outcomes are reported. The majority of studies determined seizure frequency by patient or caregiver report at clinical follow-up instead of specifying the use of a seizure diary or electroencephalography. The characterization of clinical presentation and etiology of seizures are reported in a heterogeneous manner. The rate of seizure frequency was usually measured at the last follow-up, but this ranged anywhere from 16 days to 84 months. Furthermore, there was an inconsistent presentation of antiepileptic medication at the time of treatment and whether there was a reduction or freedom from seizure medication after DBS treatment.

\section{Conclusions}

This is the first systematic review that analyzes the effectiveness of DBS on epilepsy in children and youth. Overall, the outcomes analyzed suggest promise for bilateral DBS stimulation of the ATN or CM to achieve a reduction in seizure frequency. Given the initial nascent results seen in a few patients with DBS placement in the hippocampus, STN, posteromedial hypothalamus, mammillothalamic tract, or caudal zona incerta, further understanding of the pathways may better direct the use of these anatomical targets for specifically indicated epilepsy patients. Future studies directed at blinded randomized control trials of stimulation-on/-off states of DBS after placement, with a consistent framework for the reporting of outcomes and complications, will lead to a better understanding of the best clinical scenarios to utilize DBS in children with epilepsy. This would provide an adjunct treatment option for children with DRE who are not candidates for resective surgery.

\section{References}

1. Anderson DG, Németh AH, Fawcett KA, Sims D, Miller J, Krause A: Deep brain stimulation in three related cases of North Sea progressive myoclonic epilepsy from South Africa. Mov Disord Clin Pract 4:249-253, 2017

2. Benabid AL, Minotti L, Koudsié A, de Saint Martin A, Hirsch E: Antiepileptic effect of high-frequency stimulation of the subthalamic nucleus (corpus luysi) in a case of medically intractable epilepsy caused by focal dysplasia: a 30-month follow-up: technical case report. Neurosurgery 50:1385-1392, 2002

3. Benedetti-Isaac JC, Torres-Zambrano M, Vargas-Toscano A, Perea-Castro E, Alcalá-Cerra G, Furlanetti LL, et al: Seizure frequency reduction after posteromedial hypothalamus deep brain stimulation in drug-resistant epilepsy associated with intractable aggressive behavior. Epilepsia 56:1152-1161, 2015

4. Boon P, Vonck K, Van Roost D, Clayes P, De Herdt V, Achten E, et al: Amygdalohippocampal deep brain stimulation (AHDBS) for refractory temporal lobe epilepsy. Rev Neurol (Paris) 161 (Suppl 1):1S19-1S21, 2005

5. Chabardès S, Kahane P, Minotti L, Koudsie A, Hirsch E, Benabid AL: Deep brain stimulation in epilepsy with particular reference to the subthalamic nucleus. Epileptic Disord 4 (Suppl 3):S83-S93, 2002

6. Cukiert A, Cukiert CM, Burattini JA, Lima AM: Seizure outcome after hippocampal deep brain stimulation in a prospective cohort of patients with refractory temporal lobe epilepsy. Seizure 23:6-9, 2014

7. Cukiert A, Cukiert CM, Burattini JA, Mariani PP, Bezerra DF: Seizure outcome after hippocampal deep brain stimulation in patients with refractory temporal lobe epilepsy: a prospective, controlled, randomized, double-blind study. Epilepsia 58:1728-1733, 2017

8. Cukiert A, Lehtimäki K: Deep brain stimulation targeting in refractory epilepsy. Epilepsia 58 (Suppl 1):80-84, 2017 
9. Ding P, Zhang S, Zhang J, Hu X, Yu X, Liang S, et al: Contralateral hippocampal stimulation for failed unilateral anterior temporal lobectomy in patients with bilateral temporal lobe epilepsy. Stereotact Funct Neurosurg 94:327-335, 2016

10. Dwivedi R, Ramanujam B, Chandra PS, Sapra S, Gulati S, Kalaivani M, et al: Surgery for drug-resistant epilepsy in children. N Engl J Med 377:1639-1647, 2017

11. Engel J Jr, McDermott MP, Wiebe S, Langfitt JT, Stern JM, Dewar S, et al: Early surgical therapy for drug-resistant temporal lobe epilepsy: a randomized trial. JAMA 307:922-930, 2012

12. Fisher R, Salanova V, Witt T, Worth R, Henry T, Gross R, et al: Electrical stimulation of the anterior nucleus of thalamus for treatment of refractory epilepsy. Epilepsia 51:899-908, 2010

13. Fisher RS, Uematsu S, Krauss GL, Cysyk BJ, McPherson R, Lesser RP, et al: Placebo-controlled pilot study of centromedian thalamic stimulation in treatment of intractable seizures. Epilepsia 33:841-851, 1992

14. Geller EB, Skarpaas TL, Gross RE, Goodman RR, Barkley GL, Bazil CW, et al: Brain-responsive neurostimulation in patients with medically intractable mesial temporal lobe epilepsy. Epilepsia 58:994-1004, 2017

15. Gibson WS, Ross EK, Han SR, Van Gompel JJ, Min HK, Lee $\mathrm{KH}$ : Anterior thalamic deep brain stimulation: functional activation patterns in a large animal model. Brain Stimul 9:770-773, 2016

16. Ibrahim GM, Rutka JT, Snead OC III: Epilepsy surgery in childhood: no longer the treatment of last resort. CMAJ 186:973-974, 2014

17. Jobst BC, Kapur R, Barkley GL, Bazil CW, Berg MJ, Bergey $\mathrm{GK}$, et al: Brain-responsive neurostimulation in patients with medically intractable seizures arising from eloquent and other neocortical areas. Epilepsia 58:1005-1014, 2017

18. Khan S, Wright I, Javed S, Sharples P, Jardine P, Carter M, et al: High frequency stimulation of the mamillothalamic tract for the treatment of resistant seizures associated with hypothalamic hamartoma. Epilepsia 50:1608-1611, 2009

19. Kim SH, Lim SC, Yang DW, Cho JH, Son BC, Kim J, et al: Thalamo-cortical network underlying deep brain stimulation of centromedian thalamic nuclei in intractable epilepsy: a multimodal imaging analysis. Neuropsychiatr Dis Treat 13:2607-2619, 2017

20. Kokoszka MA, Panov F, La Vega-Talbott M, McGoldrick PE, Wolf SM, Ghatan S: Treatment of medically refractory seizures with responsive neurostimulation: 2 pediatric cases. J Neurosurg Pediatr 21:421-427, 2018

21. Krishna V, King NKK, Sammartino F, Strauss I, Andrade DM, Wennberg RA, et al: Anterior nucleus deep brain stimulation for refractory epilepsy: insights into patterns of seizure control and efficacious target. Neurosurgery 78:802-811, 2016

22. Kwan P, Arzimanoglou A, Berg AT, Brodie MJ, Allen Hauser W, Mathern G, et al: Definition of drug resistant epilepsy: consensus proposal by the ad hoc Task Force of the ILAE Commission on Therapeutic Strategies. Epilepsia 51:10691077,2010

23. Laxpati NG, Kasoff WS, Gross RE: Deep brain stimulation for the treatment of epilepsy: circuits, targets, and trials. Neurotherapeutics 11:508-526, 2014

24. Lee CY, Lim SN, Wu T, Lee ST: Successful treatment of refractory status epilepticus using anterior thalamic nuclei deep brain stimulation. World Neurosurg 99:14-18, 2017

25. Lee KJ, Jang KS, Shon YM: Chronic deep brain stimulation of subthalamic and anterior thalamic nuclei for controlling refractory partial epilepsy. Acta Neurochir Suppl 99:87-91, 2006

26. Lee KJ, Shon YM, Cho CB: Long-term outcome of anterior thalamic nucleus stimulation for intractable epilepsy. Stereotact Funct Neurosurg 90:379-385, 2012

27. Lee WG, Shon YM, Seo DW: Electrical stimulation of the anterior nucleus of the thalamus for the treatment of intractable epilepsy: a longitudinal data analysis. Epilepsia 57 (Suppl 2):39, 2016 (Abstract)

28. Liberati A, Altman DG, Tetzlaff J, Mulrow C, Gøtzsche PC, Ioannidis JPA, et al: The PRISMA statement for reporting systematic reviews and meta-analyses of studies that evaluate health care interventions: explanation and elaboration. PLoS Med 6:e1000100, 2009

29. Lim SN, Lee ST, Tsai YT, Chen IA, Tu PH, Chen JL, et al: Electrical stimulation of the anterior nucleus of the thalamus for intractable epilepsy: a long-term follow-up study. Epilepsia 48:342-347, 2007

30. Lipsman N, Ellis M, Lozano AM: Current and future indications for deep brain stimulation in pediatric populations. Neurosurg Focus 29(2):E2, 2010

31. Morris GL III, Mueller WM: Long-term treatment with vagus nerve stimulation in patients with refractory epilepsy. The Vagus Nerve Stimulation Study Group E01-E05. Neurology 53:1731-1735, 1999

32. Möttönen T, Katisko J, Haapasalo J, Tähtinen T, Kiekara T, Kähärä V, et al: Defining the anterior nucleus of the thalamus (ANT) as a deep brain stimulation target in refractory epilepsy: Delineation using 3 T MRI and intraoperative microelectrode recording. Neuroimage Clin 7:823-829, 2015

33. Oh YS, Kim HJ, Lee KJ, Kim YI, Lim SC, Shon YM: Cognitive improvement after long-term electrical stimulation of bilateral anterior thalamic nucleus in refractory epilepsy patients. Seizure 21:183-187, 2012

34. Oikawa H, Sasaki M, Tamakawa Y, Kamei A: The circuit of Papez in mesial temporal sclerosis: MRI. Neuroradiology 43:205-210, 2001

35. Salanova V, Fisher R: Long term efficacy of the SANTE trial (Stimulation of the Anterior Nucleus of Thalamus for Epilepsy). Epilepsy Curr 13 (Suppl 1):123-124, 2013 (Abstract)

36. Smyth MD, Tubbs RS, Bebin EM, Grabb PA, Blount JP: Complications of chronic vagus nerve stimulation for epilepsy in children. J Neurosurg 99:500-503, 2003

37. Valentín A, García Navarrete E, Chelvarajah R, Torres C, Navas M, Vico L, et al: Deep brain stimulation of the centromedian thalamic nucleus for the treatment of generalized and frontal epilepsies. Epilepsia 54:1823-1833, 2013

38. Valentín A, Selway RP, Amarouche M, Mundil N, Ughratdar I, Ayoubian L, et al: Intracranial stimulation for children with epilepsy. Eur J Paediatr Neurol 21:223-231, 2017

39. van Rijckevorsel K, Abu Serieh B, de Tourtchaninoff M, Raftopoulos Cl: Deep EEG recordings of the mammillary body in epilepsy patients. Epilepsia 46:781-785, 2005

40. Velasco AL, Velasco F, Jiménez F, Velasco M, Castro G, Carrillo-Ruiz JD, et al: Neuromodulation of the centromedian thalamic nuclei in the treatment of generalized seizures and the improvement of the quality of life in patients with Lennox-Gastaut syndrome. Epilepsia 47:1203-1212, 2006

41. Velasco AL, Velasco M, Velasco F, Menes D, Gordon F, Rocha L, et al: Subacute and chronic electrical stimulation of the hippocampus on intractable temporal lobe seizures: preliminary report. Arch Med Res 31:316-328, 2000

42. Velasco AL, Velasco F, Velasco M, Trejo D, Castro G, Carrillo-Ruiz JD: Electrical stimulation of the hippocampal epileptic foci for seizure control: a double-blind, long-term follow-up study. Epilepsia 48:1895-1903, 2007

43. Velasco F, Velasco AL, Velasco M, Jiménez F, Carrillo-Ruiz JD, Castro G: Deep brain stimulation for treatment of the epilepsies: the centromedian thalamic target. Acta Neurochir Suppl 97 (Pt 2):337-342, 2007

44. Velasco F, Velasco M, Jiménez F, Velasco AL, Brito F, Rise $\mathrm{M}$, et al: Predictors in the treatment of difficult-to-control 
seizures by electrical stimulation of the centromedian thalamic nucleus. Neurosurgery 47:295-305, 2000

45. Velasco F, Velasco M, Ogarrio C, Fanghanel G: Electrical stimulation of the centromedian thalamic nucleus in the treatment of convulsive seizures: a preliminary report. Epilepsia 28:421-430, 1987

\section{Disclosures}

Dr. Kalia: speaker's honorarium from Medtronic.

\section{Author Contributions}

Conception and design: Ibrahim. Acquisition of data: Yan, Toyota, Anderson. Analysis and interpretation of data: Yan. Drafting the article: Yan, Toyota. Critically revising the article: all authors. Reviewed submitted version of manuscript: Yan, Anderson, Abel, Donner, Kalia, Drake, Rutka, Ibrahim. Approved the final version of the manuscript on behalf of all authors: Yan. Study supervision: Ibrahim.

\section{Correspondence}

Han Yan: The Hospital for Sick Children, Toronto, ON, Canada. hhan.yan@mail.utoronto.ca. 